\title{
EXPANSÃO URBANA E CONSERVAÇÃO DA DIVERSIDADE FLORÍSTICA: O CASO DAS Restingas da Zona de Expansão de Aracaju, SE
}

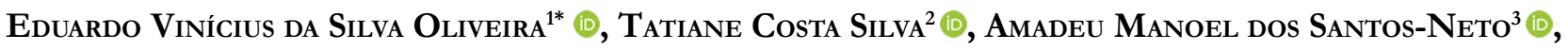 \\ Gicelia de Jesus Felix ${ }^{3}$ (อ), Myrna Friederichs Landim² ${ }^{2}$
}

1 Programa de Pós-graduação em Ecologia e Conservação, Universidade Federal de Sergipe. Cidade Univ. Prof. José Aloísio de Campos. Av. Marechal Rondon, $s / n$, Jd. Rosa Elze. São Cristóvão, Sergipe, Brasil. CEP 49100-000.

2 Departamento de Biologia, Universidade Federal de Sergipe. Cidade Univ. Prof. José Aloísio de Campos. Av. Marechal Rondon, s/n, Jd. Rosa Elze. São Cristóvão, Sergipe, Brasil. CEP 49100-000.

3 Departamento de Ecologia, Universidade Federal de Sergipe. Cidade Univ. Prof. José Aloísio de Campos. Av. Marechal Rondon, s/n, Jd. Rosa Elze. São Cristóvão, Sergipe, Brasil. CEP 49100-000.

* Autor correspondente: eduardovso@yahoo.com.br

Recebido em 27 de novembro de 2019. Aceito em 06 de julho de 2020. Publicado em 31 de julho de 2020.

Resumo - Embora as Restingas possuam considerável biodiversidade, elas estão entre os ecossistemas mais ameaçados do domínio de Mata Atlântica devido a sua localização nas áreas de ocupação e expansão urbana no litoral brasileiro. Neste estudo, foi realizada uma caracterização das Restingas da Zona de Expansão de Aracaju, estado de Sergipe. Uma lista florística foi elaborada e as fitofisionomias foram identificadas a partir de incursões a campo, complementadas pela compilação de dados de coletas botânicas prévias. Foram, ainda, realizadas análises de similaridade florística e distinção taxonômica entre as Restingas de Aracaju e outras áreas de Restinga da região Nordeste. Foram encontradas 181 espécies (sendo duas destas exóticas invasoras), distribuídas em seis fitofisionomias. As famílias de maior riqueza específica foram Fabaceae (34 espécies), Cyperaceae (23 espécies) e Rubiaceae (12 espécies). O baixo valor de distinção taxonômica registrado para as Restingas estudadas foi provavelmente devido à presença de impactos antrópicos observados in situ. A formação de grupos floristicamente similares foi associada à distância geográfica, embora não sejam descartadas hipóteses ecológicas e históricas. Ressalta-se a necessidade de ações visando à preservação dessa região, como o estabelecimento de unidades de conservação, uma clara delimitação das áreas de preservação permanente e uma mais efetiva fiscalização dessa região.

Palavras-chave: Fitofisionomias; Similaridade Florística; Distinção Taxonômica; Planícies Costeiras.

URBAN EXPANSION AND CONSERVATION OF FLORISTIC DIVERSITY: THE CASE OF THE RESTINGAS FROM THE EXPANSION ZONE of Aracaju, Sergipe state, Brazil

Aвstract - Although the Restingas (coastal vegetation on sandy soils) present considerable biodiversity, they are among the most threatened ecosystems of the Brazilian Atlantic Rainforest domain. In this study, we characterize the Restingas of a region under urban expansion, in Aracaju, Sergipe, Northeastern Brazil. We prepared a floristic list and the phytophysiognomies were identified from field surveys, complemented by data derived from previous botanical sampling in the region. Subsequently, we performed analyses of similarity and taxonomic distinctness between the Restingas of Aracaju and other five Restinga sites of Northeastern Brazil. 181 species were found (two of which are alien species) within six distinct Restinga phytophysiognomies. The most speciose families were Fabaceae (34 species), Cyperaceae (23 species), and Rubiaceae (12 species). We recorded a low value of taxonomic distinctness between these Restingas, possibly due to the presence of anthropic impacts observed in situ. The formation of floristically similar groups between the six Restingas analyzed was associated with geographic distance, although ecological and historical hypotheses could not be ruled out. We emphasize the need for actions for the preservation of the natural habitats of this region, such as the establishment of conservation units, a clear delimitation of the boundaries of permanent preservation areas and a more effective inspection by environmental agencies. 
KeYwords: Phytophysiognomies; Floristic similarity; Taxonomic distinctness; Coastal Plains.

EXPANSIÓN URBANA Y CONSERVACIÓN DE LA DIVERSIDAD FLORÍSTICA: EL CASO DE LAS RESTINGAS DE LA “ZONA DE EXPANSIÓN" de Aracaju, estado de Sergipe, Brasil

Resumen - Aunque las Restingas (Vegetación costera) poseen una biodiversidad considerable, se encuentran entre los ecosistemas más amenazados en el dominio de la Selva Atlántica debido a su ubicación en las áreas de ocupación y expansión urbana en el litoral brasileño. En este estudio, se realizó una caracterización de las Restingas de una región de la ciudad de Aracaju (llamada "Zona de Expansión”), en el estado de Sergipe a noreste de Brasil. Se elaboró una lista florística y se identificaron las fitofisionomias a partir de incursiones de campo, complementadas por la recopilación de datos de recolecciones botánicas anteriores. También se realizaron análisis de similitud florística y distinción taxonómica entre las Restingas de Aracaju y otras áreas de Restinga de la región noreste. Se encontraron 181 especies (dos de ellas exóticas invasoras), distribuidas en seis fitofisionomias. Las familias con mayor riqueza específica fueron Fabaceae (34 especies), Cyperaceae (23 especies) y Rubiaceae (12 especies). El bajo valor de distinción taxonómica registrado para las Restingas estudiadas probablemente se deba a la presencia de impactos antrópicos observados "in situ". La formación de grupos florísticamente similares se asoció con la distancia geográfica, aunque no se descartan las hipótesis ecológicas e históricas. Se destaca la necesidad de acciones dirigidas a la preservación de esta región, como el establecimiento de unidades de conservación, una delimitación clara de las áreas de preservación permanente y una inspección más efectiva de esta región.

Palabras Clave: Fitofisionomoas; Similitud florística; Distinción taxonómica; llanuras costeras.

\section{INTRODUÇÃO}

A costa brasileira, com aproximadamente 9,2 mil $\mathrm{km}$ de extensão, possuí considerável heterogeneidade geomorfológica, incluindo a Formação Barreiras, de origem Terciária, Planícies Costeiras Arenosas, de origem Quaternária, e Afloramentos Rochosos do Pré-Cambriano (Villwock et al. 2005).

Originadas por regressões marinhas, as Planícies Costeiras Arenosas são formadas por depósitos litorâneos, com sedimentação atribuída à: (i) disponibilidade de sedimentos; (ii) correntes de deriva litorânea, paralelas a costa e originada por incidência obliqua das ondas na praia; (iii) flutuações do nível do mar; e (iv) feições costeiras propiciando a retenção de sedimentos (Suguio e Tessler 1984; Villwock et al. 2005). Nestas Planícies, as areias são constantemente remobilizadas pelo vento, sendo que, em alguns locais, grandes volumes desse sedimento são transportados da praia e acumulados em campos de dunas (Villwock et al. 2005).

Sobre as Planícies Costeiras Arenosas brasileiras desenvolve-se um tipo vegetacional adjacente ao oceano, referido na literatura botânica ou ecológica como Restinga (Araujo e Henriques 1984; Falkenberg 1999), representando 79\% da vegetação costeira do Brasil (Lacerda et al. 1993). Sua flora possui forte relação com os solos arenosos das Planícies Litorâneas, sendo mais dependentes das condições edáficas do que climáticas (Marques et al. 2015).

A vegetação de Restinga distribui-se em mosaico, com tipologias herbáceas na região mais próxima à praia, até formações arbustivo-arbóreas, em direção ao continente (Araujo e Pereira 2009). Assim, as Restingas são constituídas por diferentes fitofisionomias, que por sua vez, são influenciadas principalmente por atributos do solo (Pinto-Sobrinho et al. 2011; Oliveira e Landim 2014). Em parte devido a essa variação espacial local e grande extensão latitudinal, as Restingas possuem elevada riqueza florística, fato observado nas regiões Norte (Amaral et al. 2008), Nordeste (Zickel et al. 2007; Santos-Filho et al. 2011; Gomes e Guedes 2014; Oliveira et al. 2014), Sudeste (Pereira e Araújo 2000; Martins et al. 2008) e Sul (Reitz 1961; 
Rambo 1954), sendo estimada, até o momento, a ocorrência de 2.610 espécies de Angiospermas nesses ambientes (BFG 2018).

As áreas de Restinga possuem elevada importância ecológica (Sacramento et al. 2007), fornecendo, além de sua contribuição para a manutenção da diversidade biológica, recursos para a fauna residente e migratória (Falkenberg 1999). Esta vegetação atua, ainda, na consolidação do substrato arenoso das Planícies Costeiras, amenizando a ação eólica sobre a paisagem (Cordazzo et al. 2006), papel particularmente relevante considerandose a crescente ocupação nas zonas costeiras (Moraes 2007).

A legislação brasileira prevê a proteção da vegetação de Restinga, por meio das leis 11.428/2006 e 12.651/2012, conhecidas, respectivamente, como "Lei da Mata Atlântica" (Brasil 2006) e "Lei de Proteção da Vegetação Nativa” (Brasil 2012). Entretanto, nesta última, a proteção é apenas considerada quando a vegetação de Restinga "atua como fixadora de dunas", configurando-se como Área de Preservação Permanente (Art. $4^{\circ}$, Inciso VI). Embora protegidas por lei, as Restingas se encontram ameaçadas devido ao grande adensamento populacional das zonas litorâneas, estando entre os ecossistemas brasileiros mais impactados (Holzer et al. 2004; Scherer et al. 2005).

Nas Restingas de Sergipe, no litoral nordestino, diversos impactos antrópicos têm sido registrados, como por exemplo, desmatamento, queimadas, pastejo, acúmulo de lixo, introdução de espécies exóticas, ocupações irregulares e trânsito de veículos (Pergentino e Landim 2014). O conhecimento sobre a vegetação de Restinga no estado tem aumentado recentemente (e.g., Oliveira et al. 2014; Oliveira e Landim 2014; Pergentino e Landim 2014; Oliveira et al. 2015; Oliveira e Landim 2016; Santos-Neto et al. 2018; Dantas et al. 2019), embora a maior parte dos estudos tenham sido realizados em áreas do litoral norte.

Em face da intensa pressão antrópica sobre as Restingas, estudos sobre a diversidade de sua flora podem servir como incentivo à sua conservação, além de subsídio para a tomada de decisões pelo poder público. Em Sergipe, a ocupação dessas áreas para empreendimentos imobiliários, principalmente, é crescente, sendo a situação particularmente grave no município de Aracaju, capital do estado, onde restam poucas áreas de vegetação nativa e publicações sobre a flora de suas Restingas são ainda inexistentes. Nesse sentido, este estudo realizou a caracterização da flora das Restingas da Zona de Expansão de Aracaju, analisando sua similaridade florística e distinção taxonômica com outras áreas de Restinga da região Nordeste e avaliando sua importância para a conservação.

\section{Material e Métodos}

Área de estudo

A Zona de Expansão Urbana de Aracaju (ZEU), área definida pela Lei Municipal 873/1982 (Aracaju 1982), está situada na porção sul deste município (Fig.1), entre as coordenadas geográficas $10^{\circ} 58^{\prime}-11^{\circ} 06^{\prime} \mathrm{S}$ e $37^{\circ} 02^{\prime}$ $37^{\circ} 12^{\prime} \mathrm{W}$ (Oliveira e Andrade 2015). Encontra-se delimitada, ao norte, pelos bairros Atalaia e Aeroporto, ao sul, pela desembocadura do rio Vaza-Barris, a oeste, pelo rio Santa Maria, e, a leste, pelo Oceano Atlântico (Oliveira e Andrade 2015). 
Figura 1. Zona de Expansão do município de Aracaju, estado de Sergipe. Círculos em vermelho indicam a localização dos pontos de amostragem da flora em áreas de Restinga.

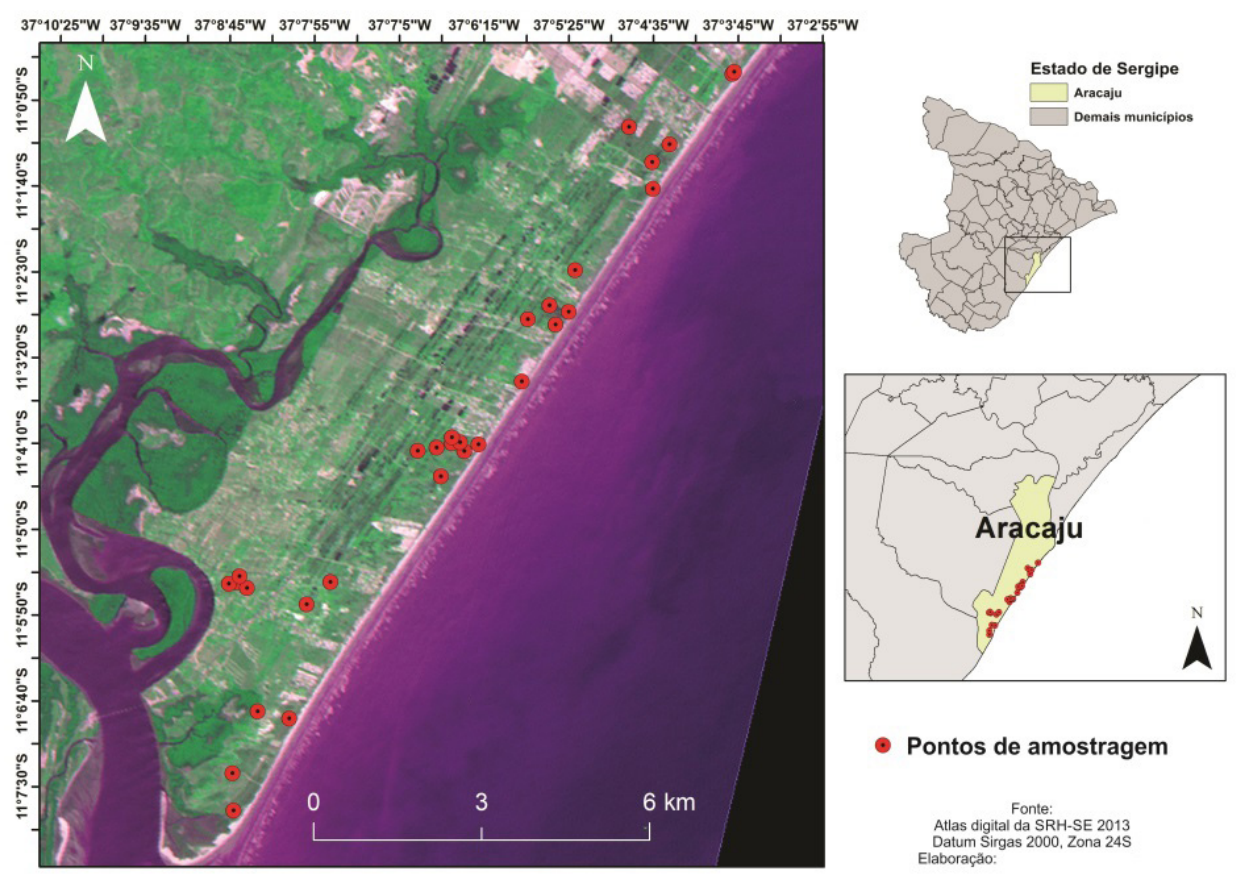

O clima da região é do tipo As, ou seja, tropical chuvoso com verão seco, de acordo com o sistema de classificação climática de Köppen-Geiger (SEPLANTEC-SE 1997; Alvares et al. 2014). A temperatura média anual é de $26^{\circ} \mathrm{C}$ e a precipitação pluviométrica média anual é de $1.590 \mathrm{~mm}$, distribuída em maior intensidade entre os meses de março a agosto (SEPLANTEC-SE 1997).

\section{Coleta e análise de dados}

Foram realizadas incursões a campo, em 28 pontos amostrais ao longo de toda a extensão da ZEU de Aracaju, durante o período de janeiro de 2012 a novembro de 2016, abrangendo os períodos seco e chuvoso de cada um desses anos.

Em todas as fitofisionomias encontradas, com base na classificação feita por Silva e Britez (2005), foram realizadas coletas de espécimes vegetais, por meio de caminhadas a procura de material fértil, o qual foi herborizado seguindo metodologia usual (Mori et al. 1985). Em seguida, o material foi depositado no Herbário da Universidade Federal de Sergipe (ASE) e identificado com base em literatura especializada (e.g., Prata et al. 2013) e por meio de comparação com outras exsicatas do acervo deste herbário, adotando-se o sistema de classificação APG IV (2016).

Para complementar as campanhas de campo, foram realizadas buscas por espécimes provenientes de coletas prévias realizadas na ZEU de Aracaju, por meio de consulta à plataforma do sistema SpeciesLink no mês de janeiro de 2017 (CRIA 2017). Foram mantidos apenas os registros cujas exsicatas estavam depositadas no acervo do Herbário ASE, de modo a possibilitar a confirmação ou atualização de sua identificação taxonômica, e eliminados aqueles provenientes de coletas muito antigas ( $<$ ano 2000), evitando assim, um viés temporal nos dados (Meyer et al. 2016). Considerando que, desde a década de 70, ocorre uma intensa destruição de áreas naturais na ZEU de Aracaju (Oliveira e Andrade 2012), adotamos este critério na tentativa de incluir apenas áreas de Restinga ainda existentes e em condições de integridade que permitam a manutenção de, ao menos, parte de sua biodiversidade. Os registros mais antigos eliminados foram coletados entre 1974 e 1983, compreendendo 
cerca de 20\% dos registros totais. Em seguida, todos os vouchers foram analisados no Herbário ASE, sendo removidos aqueles com identificação incompleta ou questionável.

A caracterização das formas de vida e substrato foi realizada por meio de observações em campo e das informações presentes nas etiquetas das exsicatas já depositadas no ASE, sendo estas classes padronizadas (BFG 2018). A lista final foi analisada quanto ao registro de novas ocorrências para as Restingas de Sergipe (Oliveira et al. 2014; 2015), espécies raras (Giulietti et al. 2009), exóticas invasoras (I3N Brasil/Instituto Hórus 2018) e ameaçadas (Martinelli e Moraes 2013).

Para a análise da similaridade florística, foram selecionadas cinco publicações abrangendo listas de espécies em áreas de Restinga em Sergipe e estados vizinhos (Alagoas e Bahia). Os critérios utilizados para a escolha destas publicações foram: 1) apresentar a mesma metodologia de coleta (método de caminhamento), 2) considerarem todos os estratos vegetais, e 3) apresentarem o levantamento da flora de uma área geograficamente restrita (não se tratando de checklists abrangendo áreas de vários municípios). Em Sergipe e Alagoas só foram encontrados um estudo, em cada estado, que atendesse a esses pré-requisitos (Oliveira et al. 2015 e Almeida-Jr et al. 2016, respectivamente). $\mathrm{Na}$ Bahia, considerando a maior extensão de sua linha de costa, foram sorteadas três publicações que atendessem a esses critérios, um para cada região de seu litoral: norte (Queiroz et al. 2012), centro (Brito et al. 1993) e sul (Fernandes e Queiroz 2015).

Após a escolha das publicações, foi realizada a padronização dos nomes das plantas listadas nestes estudos com o uso da ferramenta Taxonomic Name Resolution Service v 4.0, mantendo apenas a única melhor combinação para cada nome (Boyle et al. 2013). Em seguida, utilizando o índice de Sørensen, foi gerado um dendograma a partir de uma matriz binária (presença/ausência) utilizando a média aritmética não ponderada por grupo (UPGMA) no aplicativo Past 2.17 (Hammer et al. 2013).

A relação entre a dissimilaridade florística e a distância espacial entre as áreas de Restinga analisadas foi avaliada por meio do teste de Mantel, usando o índice de correlação de Pearson e, posteriormente, o teste de Monte Carlo com 5.000 permutações aleatórias $(\alpha=0,05)$ no aplicativo Past 2.17 (Zar 2010; Hammer et al. 2013). A matriz de distância linear entre as áreas de Restinga analisadas foi construída no aplicativo ArcGIS 9.3 (ESRI 2008).

Por fim, a distinção taxonômica média $\left(\Delta^{+}\right)$foi calculada utilizando os dados das publicações analisadas acima, por meio do aplicativo Past 2.17 (Hammer et al. 2013). Em um sentido filogenético, a distinção taxonômica está baseada na ideia de que comunidades estreitamente relacionadas possuem menor diversidade do que comunidades com pouca relação entre suas espécies (Moreno et al. 2009). Para essa análise, foram utilizados os níveis taxonômicos de ordem, família, gênero e espécie, adotando o mesmo peso (=1) para as distâncias entre os diferentes níveis (Warwick e Clarke 1995).

\section{RESUltados}

Ao todo, foram encontradas 181 espécies [sendo 132 (73\%) delas oriundas das coletas de campo realizadas pelos autores], pertencentes a 131 gêneros e 61 famílias (Tab. 1). 
Tabela 1. Angiospermas registradas em áreas de Restinga da Zona de Expansão do município de Aracaju, estado de Sergipe. + = novo registro de ocorrência para as Restingas de Sergipe; * = espécie exótica invasora; $\mathrm{FV} / \mathrm{S}=$ forma de vida/substrato; $\mathbf{E R}=$ erva; $\mathrm{SA}=$ subarbusto; $\mathrm{AR}=$ arbusto; $\mathbf{A}$ = árvore; $\mathrm{LVT}=\mathrm{Liana} /$ volúvel/ trepadeira; $\mathbf{P A}=$ palmeira; $\mathrm{SU}=$ suculenta; $\mathrm{T}=$ terrícola; $\mathrm{A}=$ aquático; $\mathrm{HE}=$ hemiepífita; $\mathrm{HP}=$ hemiparasita; $\mathrm{R}$ = rupícola.

Família/Espécie

Amaranthaceae

Alternanthera tenella Colla +

Anacardiaceae

Anacardium occidentale $\mathrm{L}$.

Scbinus terebinthifolius Raddi (Figura 2c)

Apocynaceae

Matelea ganglinosa (Vell.) Rapini +

Araceae

Antburium affine Schott

Philodendron imbe Schott ex Kunth.

Araliaceae

Hydrocotyle bonariensis Lam.

Arecaceae

Allagoptera campestris (Mart.) Kuntze

Syagrus coronata (Mart.) Becc.

Syagrus schizopbylla (Mart.) Glassman

Aristolochiaceae

Aristolocbia birostris Duch.

Asteraceae

Acmella sp. ${ }^{+}$

Centratherum punctatum Cass. +

Conocliniopsis prasiifolia (DC.) R.M.King \& H.Rob

Eclipta prostrata (L.) L.

Emilia fosbergii Nicolson +

Lepidaploa mucronifolia (DC.) H.Rob. (Figura 2a)

Praxelis clematidea (Griseb.) R.M.King \& H.Rob. +

Tilesia baccata (L.f.) Pruski

Vernonia sp.

Boraginaceae

Euploca polyphylla (Lehm.) J.I.M.Melo \&Semir

Heliotropium sp.

Bromeliaceae

Aechmea sp.

Hohenbergia catingae Ule
$\mathrm{ER} / \mathrm{T}$

FV/S Coletor e n ${ }^{\circ}$ (Herbário ASE)

SA/T Oliveira, E.V.S. 60

$\mathrm{A} / \mathrm{T}$

Oliveira, E.V.S. 53

$\mathrm{A} / \mathrm{T}$

Oliveira, E.V.S. 86

LVT/T

Oliveira, E.V.S. 480

$\mathrm{ER} / \mathrm{R}$

Silva, T.C. A19

LVT/HE

Oliveira, E.V.S. 38

ER/T

Oliveira, E.V.S. 1

ER/T

Landim, M.F. 1522

$\mathrm{PA} / \mathrm{T}$

Oliveira, E.V.S. 54

$\mathrm{PA} / \mathrm{T}$

Oliveira, E.V.S. 614

LVT/T

Oliveira, E.V.S. 613

ER/T

Farias, M.C.V. 718

SA/T

Farias, M.C.V. 653

SA/T

Oliveira, E.V.S. 31

$\mathrm{ER} / \mathrm{T}$

Oliveira, E.V.S. 70

ER/T

$\mathrm{SA} / \mathrm{T}$

Santos, E. 166

SA/T

Oliveira, E. V. S.

$\mathrm{SA} / \mathrm{T}$

Santos, E. 165

ER/T

Oliveira, E.V.S.

Landim, M.F.1487

ER/T

Oliveira, E. V. S.

ER/T

Figueiredo, M.A. 11

$\mathrm{ER} / \mathrm{T}$
Silva, T.C. A51

Oliveira, E.V.S. 52A 
Família/Espécie

\section{Cactaceae}

Cereus fernambucensis Lem. subsp. fernambucensis

Pilosocerens catingicola subsp. Salvadorensis (Werderm.) Zappi (Figura 21)

\section{Capparaceae}

Cynophalla flexuosa (L.) J.Presl (Figura 2j)

\section{Chrysobalaceae}

Chrysobalanus icaco $\mathrm{L}$.

\section{Combretaceae}

Conocarpus erectus $\mathrm{L}$.

Laguncularia racemosa (L.) C.F.Gaertn.

\section{Commelinaceae}

Commelina erecta $\mathrm{L}$.

\section{Convolvulaceae}

Daustinia montana (Moric.) Buril \& A.R. Simões

Ipomoea asarifolia (Desr.) Roem. \&Schult.

Ipomoea imperati (Vahl) Griseb.

Ipomoea pes-caprae (L.) R.Br. (Figura 2h)

Jacquemontia evolvuloides (Moric.) Meisn.

\section{Cyperaceae}

Bulbostylis junciformis(Kunth) C.B.Clarke

Cyperus articulatus $\mathrm{L}$.

Cyperus compressus $\mathrm{L}$.

Cyperus crassipes Vahl

Cyperus haspan $\mathrm{L}$.

Cyperus ligularis L. (Figura 2b)

Cyperus squarrosus $\mathrm{L}$.

Cyperus surinamensis Rottb.

Eleocharis endounifascis Hinchliff \& Roalson +

Eleocharis geniculata (L.) Roem. \&Schult.

Fimbristylis complanata (Retz.) Link

Fimbristylis cymosa R.Br.

Fimbristylis dichotoma (L.) Vahl

Fimbristylis ferruginea (L.) Vahl

Fuirena umbellata Rottb.

Kyllinga brevifolia Rottb

Kyllinga squamulata Thonn. ex Vahl

Kyllinga vaginata Lam.

Lipocarpha micrantha (Vahl) G.C.Tucker

Pycreus polystachyos (Rottb.) P.Beauv.
$\mathrm{FV} / \mathrm{S}$

Coletor e $\mathrm{n}^{\circ}$ (Herbário ASE)

$\mathrm{SU} / \mathrm{T}$

Oliveira, E.V.S. 20

$\mathrm{SU} / \mathrm{T}$

Oliveira, E.V.S. 80

$\mathrm{AR} / \mathrm{T}$

Oliveira, E.V.S. 611

$\mathrm{AR} / \mathrm{T}$

Oliveira, E.V.S. 6

$\mathrm{A} / \mathrm{T}$

Oliveira, E.V.S. 4

$\mathrm{A} / \mathrm{T}$

Oliveira, E.V.S. 5

$\mathrm{ER} / \mathrm{T}$

Oliveira, E.V.S. 57

LVT/T

Oliveira, E.V.S. 475

LVT/T

Farias, M.C.V. 650

LVT/T

Oliveira, E.V.S. 77

LVT/T

Oliveira, E.V.S. 64

$\mathrm{ER} / \mathrm{T}$

Oliveira, E.V.S. 56

ER/T

Silva 140

ER/A

ER/T

Menezes, I.R.N. 218

ER/T

Silva 130

ER/T

ER/T

ER/T

ER/T

ER/A

ER/A

ER/T

ER/T

$\mathrm{ER} / \mathrm{T}$

ER/T

ER/A

ER/T

ER/T

ER/T

ER/T

$\mathrm{ER} / \mathrm{T}$
Landim, M.F. 1524

Menezes, I.R.N. 214

Oliveira, E.V.S. 18

Silva 121

Menezes, I.R.N. 216

Menezes, I.R.N. 208

Oliveira, E.V.S. 604

Silva 135

Menezes, I.R.N. 211

Silva, T.C. 12

Costa, S.M. 168

Menezes, I.R.N. 206

Silva 126

Costa, S.M. 163

Menezes, I.R.N. 213

Menezes, I.R.N. 217

Silva, T.C. 57 
Família/Espécie

Rhynchospora ciliata (Vahl) Kük. +

Rhynchospora riparia (Nees) Boeckeler

Scleria hirtella Sw.

Dilleniaceae

Tetracera breyniana Schltdl. (Figura 2g)

\section{Eriocaulaceae}

Paepalanthus tortilis (Bong.) Mart.

\section{Erythroxylaceae}

Erythroxylum passerinum Mart.

\section{Euphorbiaceae}

Acalypha sp. +

Sapium argutum (Müll.Arg.) Huber +

\section{Fabaceae}

Aeschynomene histrix Poir.

Aeschynomene montevidensis Vogel +

Aeschynomene viscidula Michx.

Andira fraxinifolia Benth

Canavalia brasiliensis Mart. ex Benth. +

Canavalia rosea (Sw.) DC.

Centrosema brasilianum (L.) Benth. (Figura 2f)

Centrosema pascuorum Mart. ex Benth.

Chamaecrista amiciella (H.S.Irwin \& Barneby) H.S.Irwin \& Barneby

Chamaecrista flexuosa (L.) Greene.

Chamaecrista hispidula (Vahl) H.S.Irwin \& Barneby

Chamaecrista ramosa (Vogel) H.S.Irwin \& Barneby

Clitoria laurifolia Poir.

Crotalaria pallida Aiton

Crotalaria retusa $\mathrm{L}$.

Dalbergia ecastaphyllum (L.) Taub.

Desmodium adscendens (Sw.) DC. +

Desmodium barbatum (L.) Benth.

Indigofera sabulicola Benth.

Inga ciliata C.Presl

Inga maritima Benth.

Macroptilium latbyroides (L.) Urb.

Macroptilium panduratum (Mart. ex Benth.) Maréchal \& Baudet

Mimosa arenosa (Willd.) Poir. +

Mimosa candollei R.Grether

Mimosa pudica L.
FV/S

ER/T

ER/T

ER/T

LVT/T

ER/A

A/T

ER/T

A/T

SA/T

AR/T

ER/T

A/T

LVT/T

LVT/T

LVT/T

LVT/T

SA/T

$\mathrm{SA} / \mathrm{T}$

SA/T

SA/T

ER/T

SA/T

ER/T

AR/T

SA/T

SA/T

ER/T

A/T

A/T

SA/T

LVT/T

A/T

ER/T

ER/T
Oliveira, E.V.S. 19

Menezes, I.R.N. 235

Oliveira, E.V.S. 479

Silva 122

Figueiredo, M.A. 5

Menezes, I.R.N. 212

Oliveira, E.V.S. 379

Oliveira, E.V.S. 25

Oliveira, E.V.S. 382

Silva, T.C. 10

Silva, T.C. 22

Oliveira, E.V.S. 386

Oliveira, E.V.S. 608

Oliveira, E.V.S. 154

Oliveira, E.V.S. 618

Silva, T.C. 1

Landim, M.F. 1488

Oliveira, E.V.S. 390

Oliveira, E.V.S. 14

Oliveira, E.V.S. 7

Oliveira, E.V.S. 16

Oliveira, E.V.S. 606

Almeida, C. 16

Oliveira, E.V.S. 69

Figueiredo, M.A. 19

Oliveira, E.V.S. 21

Silva, T.C. 9

Oliveira, E.V.S. 612

Oliveira, E.V.S. 46

Oliveira, E.V.S. 2

Silva, T.C. 20

Oliveira, E.V.S. 478

Silva, T.C. 23

Oliveira, E.V.S. 49 


\section{Família/Espécie}

Periandra sp.

Rhynchosia minima (L.) DC. +

Rhynchosia phaseoloides (Sw.) DC.

Senna alata (L.) Roxb. +

Senna occidentalis (L.) Link

Sesbania exasperata Kunth +

Sesbania virgata (Cav.) Pers.

Stylosanthes viscosa (L.) Sw.

Gentianaceae

Coutoubea spicata Aubl.

Schultesia guianensis (Aubl.) Malme var. guianensis

\section{Humiriaceae}

Humiria balsamifera (Aubl.) A.St.-Hil.

\section{Hydroleaceae}

Hydrolea spinosa L. (Figura 2k)

\section{Juncaceae}

Indeterminado +

Lamiaceae

Hypenia salzmannii (Benth.) Harley

Leonotis nepetifolia (L.) R.Br. +

Marsypianthes chamaedrys (Vahl) Kuntze

Rhapbiodon ecbinus (Nees\& Mart.) Schauer

Lauraceae

Cassytha filiformis $\mathrm{L}$.

LVT/HP

Lentibulariaceae

Utricularia gibba $\mathrm{L}$.

\section{Lythraceae}

Cuphea flava Spreng.

\section{Malpighiaceae}

Byrsonima gardneriana A. Juss.

\section{Malvaceae}

Pavonia bumifusa A.St.-Hil.

Sida cordifolia L. +

Waltheria albicans Turcz. +

\section{Melastomataceae}

Mouriri guianensis Aubl.

Menyanthaceae

Nymphoides humboldtiana (Kunh) Kuntze * (Figura 2d)
$\mathrm{FV} / \mathrm{S}$

Coletor e $\mathrm{n}^{\circ}$ (Herbário ASE)

AR/T

Landim, M.F. 1516

LVT/T

Farias, M.C.V. 657

LVT/T

Landim, M.F. 1499

$\mathrm{AR} / \mathrm{T}$

Farias, M.C.V. 658

$\mathrm{AR} / \mathrm{T}$

Silva, T.C. 54

AR/T

Farias, M.C.V. 717

AR/T

Oliveira, E.V.S. 469

SA/T

Landim, M.F. 1484

SA/T

Braguini, C.R. 1

ER/T

Silva, T.C. 11

$\mathrm{A} / \mathrm{T}$

Silva, T.C. 45

SA/A

Oliveira, E.V.S. 392

ER/A

Silva, T.C. 34

SA/T

Oliveira, E.V.S. 470

ER/T

Oliveira, E.V.S. 471

$\mathrm{SA} / \mathrm{T}$

Oliveira, E.V.S. 700

$\mathrm{ER} / \mathrm{T}$

Silva, T.C. 17

ER/A

Menezes, I.R.N. 239

$\mathrm{ER} / \mathrm{T}$

Oliveira, E.V.S. 15

AR/T

Oliveira, E.V.S. 79

ER/T

Oliveira, E.V.S. 33

$\mathrm{SA} / \mathrm{T}$

Farias, M.C.V. 651

SA/T

Oliveira, E.V.S. 66

A/T

Oliveira, E.V.S. 59

ER/A

Silva, T.C. 13 
Família/Espécie

$\mathrm{FV} / \mathrm{S}$

Coletor e $\mathrm{n}^{\circ}$ (Herbário ASE)

Microteaceae

Microtea paniculata Moq.

$\mathrm{ER} / \mathrm{T}$

Oliveira, E.V.S. 487A

\section{Moraceae}

Ficus americana subsp. guianensis (Desv.) C.C. Berg

Oliveira, E.V.S. 50

Ficus cyclopbylla (Miq.) Miq.

Oliveira, E.V.S. 616

\section{Myrtaceae}

Eugenia astringens Cambess.

Silva, T.C. 36

Eugenia punicifolia (Kunth) DC.

$\mathrm{AR} / \mathrm{T}$

Oliveira, E.V.S. 617

Myrcia guianensis (Aubl.) DC.

\section{Nyctaginaceae}

Guapira laxa (Netto) Furlan +

Oliveira, E.V.S. 81

Guapira opposita (Vell.) Reitz

Guapira pernambucensis (Casar.) Lundell

\section{Nymphaceae}

Nymphaea sp.

\section{Olacaceae}

Ximenia americana $\mathrm{L}$.

Onagraceae

Ludwigia leptocarpa (Nutt.) H.Hara

SA/A Oliveira, E.V.S. 605

Ludwigia octovalvis (Jacq.) P.H.Raven

SA/T

Oliveira, E.V.S. 393

Papaveraceae

Argemone mexicana L. +

$\mathrm{ER} / \mathrm{T}$

Farias, M.C.V. 652

\section{Passifloraceae}

Passiflora edulis Sims

LVT/T

Silva, T.C. 33

Passiflora foetida L.

$\mathrm{LVT} / \mathrm{T}$

Oliveira, E.V.S. 23

Passiflora silvestris Vell.

\section{Plantaginaceae}

Bacopa monnieri (L.) Pennell

$\mathrm{ER} / \mathrm{A}$

Oliveira, E.V.S. 68

Scoparia dulcis L.

$\mathrm{SA} / \mathrm{T}$

Oliveira, E.V.S. 391

Plumbaginaceae

Plumbago scandens L.

$\mathrm{AR} / \mathrm{T}$

Oliveira, E.V.S. 476

\section{Poaceae}

Andropogon selloanus (Hack.) Hack.

$\mathrm{ER} / \mathrm{T}$

Oliveira, E.V.S. 11

Chloris barbata Sw.

$\mathrm{ER} / \mathrm{T}$

Menezes, I.R.N. 231

Eragrostis ciliaris (L.) R.Br

$\mathrm{ER} / \mathrm{T}$

Gymnopogon foliosus (Willd.) Nees +

$\mathrm{ER} / \mathrm{T}$

Menezes, I.R.N. 229

Megathyrsus maximus (Jacq.) B.K.Simon \& S.W.L.Jacobs*

$\mathrm{ER} / \mathrm{T}$

Souza, L.S. 1

Panicum trichoides Sw.

$\mathrm{ER} / \mathrm{T}$

Silva, T.C. 18

Paspalum divergens Döll

$\mathrm{ER} / \mathrm{T}$ 


\section{Família/Espécie}

Paspalum millegrana Schrad. ex Schult. +

Paspalum vaginatum $\mathrm{Sw}$.

Setaria parviffora (Poir.) Kerguélen

Sporobolus virginicus (L.) Kunth

Polygalaceae

Asemeia ovata (Poir.) J.F.B.Pastore \& J.R.Abbott

Asemeia violacea (Aubl.) J.F.B.Pastore \&J.R.Abbott

Polygala cyparissias A.St.-Hil. \&Moq.

Polygonaceae

Coccoloba laevis Casar.

\section{Portulaceae}

Portulaca mucronata Link. +

\section{Rubiaceae}

Borreria capitata (Ruiz \&Pav.) DC.

Borreria verticillata (L.) G.Mey.

Chomelia obtusa Cham. \&Schltdl.

Mitracarpus baturitensis Sucre +

Mitracarpus eichleri K.Schum.

Mitracarpus strigosus (Thunb.) P.L.R. Moraes, De Smedt \& Hjertson+

Oldenlandia filicaulis K.Schum.

Richardia grandiffora (Cham. \&Schltdl.) Steud.

Richardia scabra L. +

Staelia virgata (Link ex Roem. \&Schult.) K.Schum.

Tocoyena bullata (Vell.) Mart.

Tocoyena sellowiana (Cham. \&Schltdl.) K. Schum. (Figura 2i)

\section{Sapindaceae}

Paullinia elegans Cambess.

\section{Sapotaceae}

Manilkara sal₹mannii (A.DC.) H.J.Lam

Sideroxylon obtusifolium (Roem. \&Schult.) T.D.Penn.

\section{Scrophulariaceae}

Scrophularia sp.

Smilacaceae

Smilax brasiliensis Spreng.

Smilax rufescens Griseb.

\section{Solanaceae}

Solanum granulosoleprosum Dunal (Figura 2e)

Solanum stipulaceum Willd. ex Roem. \&Schult.
LVT/T

FV/S Coletor e $\mathrm{n}^{\circ}$ (Herbário ASE)

$\mathrm{ER} / \mathrm{T}$

Santos, E. 164

$\mathrm{ER} / \mathrm{T}$

Costa, S.M. 359

$\mathrm{ER} / \mathrm{T}$

Oliveira, E.V.S. 61

$\mathrm{ER} / \mathrm{T}$

Costa, S.M. 363

$\mathrm{ER} / \mathrm{T}$

Oliveira, E.V.S. 615

$\mathrm{ER} / \mathrm{T}$

Oliveira, E.V.S. 22

$\mathrm{ER} / \mathrm{T}$

Costa, S.M. 383

$\mathrm{AR} / \mathrm{T}$

Oliveira, E.V.S. 474

$\mathrm{ER} / \mathrm{T}$

Oliveira, E.V.S. 380

SA/T

Farias, M.C.V. 655

SA/T

Oliveira, E.V.S. 26

$\mathrm{AR} / \mathrm{T}$

ER/T

Oliveira, E.V.S. 601

$\mathrm{ER} / \mathrm{T}$

Silva, T.C. 14

ER/T

Oliveira, E.V.S. 153

ER/A

Landim, M.F. 1506

SA/T

Menezes, I.R.N. 240

ER/T

Oliveira, E.V.S. 24

$\mathrm{ER} / \mathrm{T}$

Landim, M.F. 1485

AR/T

Landim, M.F. 1486

$\mathrm{A} / \mathrm{T}$

Oliveira, E.V.S. 384

LVT/T

Oliveira, E.V.S. 40

A/T

$\mathrm{A} / \mathrm{T}$

Menezes, I.R.N. 223

Silva, T.C. 27

ER/T

Costa, S.M. 362

LVT/T

Matos, G.M.A. 73

Oliveira, E.V.S. 609

AR/T

Oliveira, E.V.S. 389

AR/T 
Família/Espécie

Turneraceae

Piriqueta duarteana (Cambess.) Urb. var. duarteana

Typhaceae

Typha domingensis Pers.

Urticaceae

Cecropia pachystachya Trécul

Verbenaceae

Lantana camara $\mathrm{L}$.

Stachytarpheta cayennensis (Rich.) Vahl

Indeterminado

Stachytarpheta microphylla Walp.

Violaceae

Pombalia calceolaria (L.) Paula-Souza

Xyridaceae

Xyris macrocephala Vahl +
FV/S Coletor e $\mathrm{n}^{\circ}$ (Herbário ASE)

SA/T Oliveira, E.V.S. 62

ER/A Menezes, I.R.N. 205

A/T Oliveira, E.V.S. 32

SA/T Silva, T.C. 15

SA/T Oliveira, E.V.S. 3

SA/T Oliveira, E.V.S. 48

SA/T Silva, T.C. 24

ER/T Oliveira, E.V.S. 55

ER/T Menezes, I.R.N. 236

Figura 2. Angiospermas coletadas em áreas de Restingas da Zona de Expansão do município de Aracaju, estado de Sergipe. a: Lepidaploa mucronifolia (DC.) H.Rob. b: Cyperus ligularis L. c: Schinus terebinthifolius Raddi. d: Nymphoides bumboldtiana (Kunh) Kuntze. e: Solanum granulosoleprosum Dunal. f: Centrosema brasilianum (L.) Benth. g: Tetracera breyniana Schltdl. h: Ipomoea pes-caprae (L.) R.Br. i: Tocoyena sellowiana (Cham. \&Schltdl.) K. Schum. j: Cynophalla flexuosa (L.) J.Presl. k: Hydrolea spinosa L.1: Pilosocerens catingicola subsp. Salvadorensis (Werderm.) Zappi. (Fotos: Eduardo V. S.

Oliveira e Tatiane C. Silva).

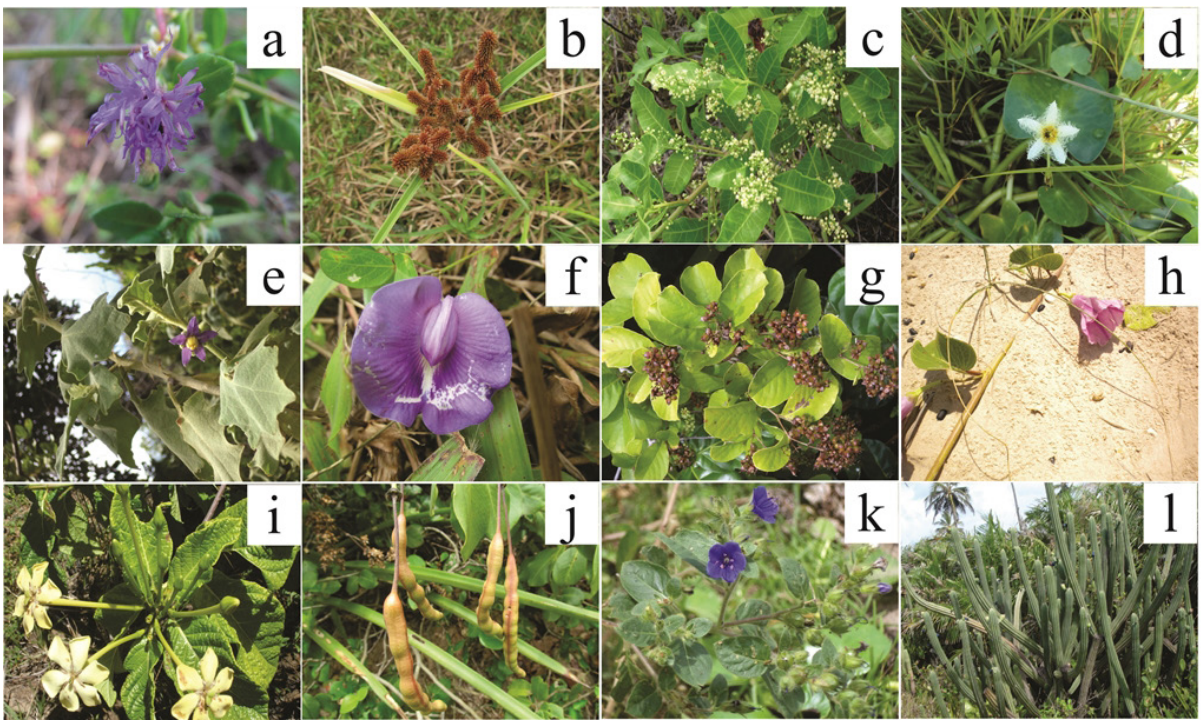

As famílias de maior riqueza específica foram Fabaceae (34 espécies), Cyperaceae (23), Rubiaceae (12), Poaceae (11) e Asteraceae (nove). Essas cinco famílias correspondem a 49\% do total de espécies, apesar de representarem apenas $8 \%$ do número total de famílias. As famílias com apenas uma espécie correspondem a $18 \%$ do total de espécies e 52\% do total de famílias (Tab. 1). Os gêneros com maior número de espécies foram Cyperus (sete), Chamaecrista e Fimbristylis (quatro, em cada). Esses três gêneros correspondem a $8 \%$ do total de espécies e $2 \%$ do total de gêneros (Tab. 1). 
Foram identificadas sete formas de vida, com predomínio das ervas (80 espécies), seguidas de subarbustos (34 espécies), árvores (24 espécies), trepadeiras e/ou lianas (22 espécies), arbustos (17 espécies), palmeiras (duas espécies) e suculentas (duas espécies).

Seis dos tipos fitofisionômicos propostos por Silva e Britez (2005), foram identificados: campo aberto não inundável (CANI), campo fechado inundável (CFI) e não inundável (CFNI), fruticeto aberto não inundável (FANI), fruticeto fechado não inundável (FFNI) e floresta não inundável (FNI) (Fig. 3). Usualmente, estas fitofisionomias distribuíram-se em mosaicos, com limites de transição entre eles pouco definidos. Em apenas um ponto amostral foi observada a fitofisionomia "floresta não inundável", sendo a maior parte da vegetação da ZEU composta por campos e fruticetos abertos.

Figura 3. Fitofisionomias registradas na Zona de Expansão do município de Aracaju, estado de Sergipe. Campo aberto não inundável (a); campo fechado inundável (b); campo fechado não inundável (c); fruticeto aberto não inundável (d) e (e); fruticeto fechado não inundável (f); e floresta fechada não inundável (g) e (h). (Fotos:

Eduardo V. S. Oliveira).

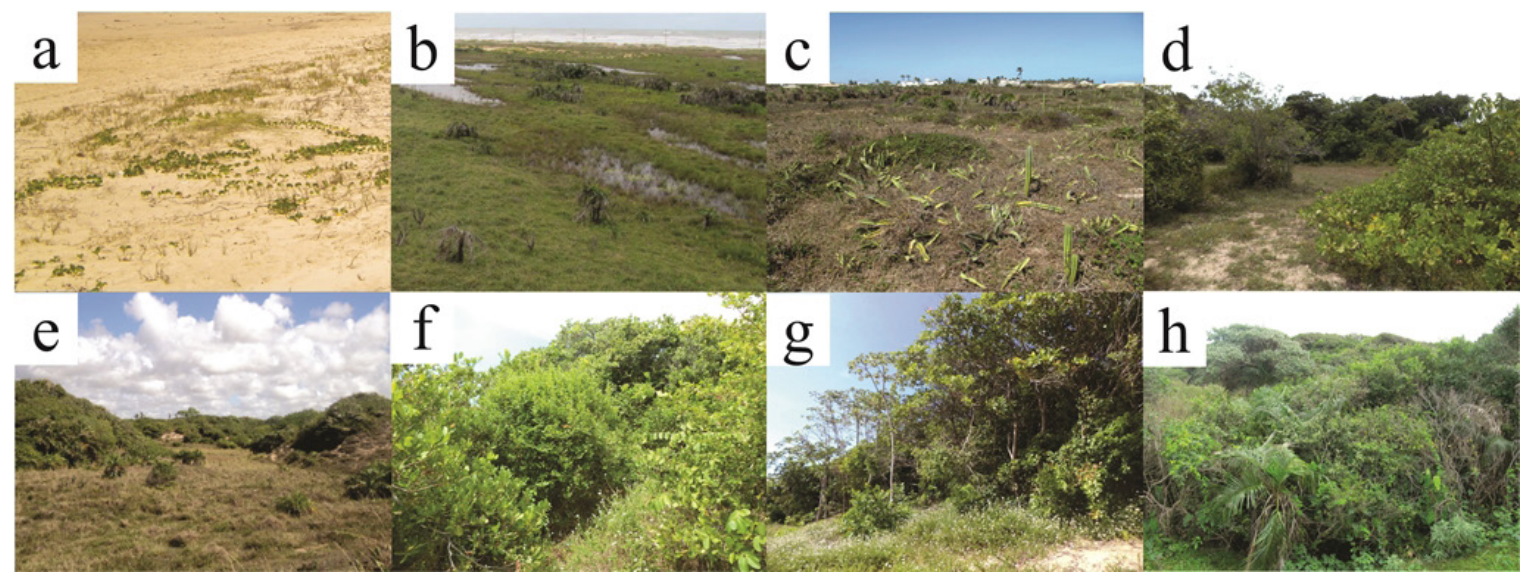

Os valores de similaridade florística entre as Restingas de Aracaju (SE1) e as demais regiões analisadas foram baixos, ou seja, inferiores a 50\%, tendo sido observados dois grupos florísticos: 1) matas do sul da Bahia (BA3); e 2) compreendendo todas as demais localidades (Fig. 4). Neste segundo grupo foram ainda identificados três subgrupos florísticos: 2.1) BA1 e BA2; 2.2) SE1 e SE2; e 2.3) AL. Observou-se, portanto, uma correlação positiva entre a dissimilaridade florística e a distância geográfica $(\mathrm{r}=0,52 ; \mathrm{p}=0,02)$.

Figura 4. Dendrograma construído a partir de uma matriz binária (presença/ausência) utilizando média aritmética não ponderada por grupo (UPGMA) a partir do índice de Sørensen, de seis levantamentos florísticos em áreas de Restinga nos estados de Alagoas, Bahia e Sergipe. AL = Almeida-Jr et al. (2016); BA1 = Queiroz et al. (2012); BA2 = Brito et al. (1993); BA3 = Fernandes e Queiroz (2015); SE1 = este estudo; SE2 = Oliveira et al. (2015).

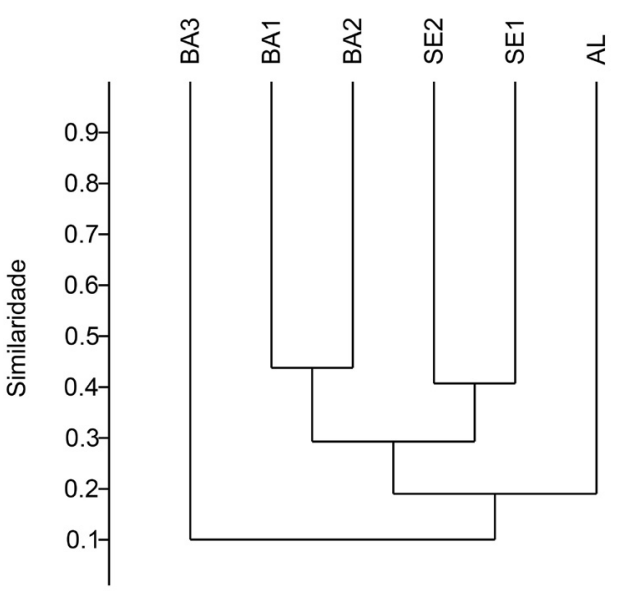


O maior valor de distinção taxonômica média foi encontrado em BA1 e o menor, na área de estudo (Tab. 2). A maior amplitude deste índice foi observada em $\operatorname{AL}(0,20)$ e a menor em $\operatorname{BA2}(0,07)$.

Tabela 2. Distinção taxonômica média $\left(\Delta^{+}\right)$e amplitude (limite inferior e superior) calculados para seis levantamentos florísticos em áreas de Restinga nos estados de Alagoas, Bahia e Sergipe. AL = Almeida-Jr et al. (2016); BA1 = Queiroz et al. (2012); BA2 = Brito et al. (1993); BA3 = Fernandes e Queiroz (2015); SE1 = este estudo; SE2 = Oliveira et al. (2015).

\begin{tabular}{lcccccc}
\hline & AL & BA1 & BA2 & BA3 & SE1 & SE2 \\
\hline$\Delta^{+}$ & 4,66 & 4,68 & 4,66 & 4,67 & 4,54 & 4,65 \\
Limite inferior & 4,56 & 4,63 & 4,64 & 4,63 & 4,61 & 4,62 \\
Limite superior & 4,77 & 4,71 & 4,70 & 4,71 & 4,72 & 4,71 \\
\hline
\end{tabular}

Do total de espécies registradas, 30 destas (16\%) são novas ocorrências para as Restingas de Sergipe. Apesar de não terem sido encontradas espécies ameaçadas e/ou raras na ZEU de Aracaju, duas espécies exóticas invasoras [Nymphoides humboldtiana (Kunh) Kuntze e Megatbyrsus maximus (Jacq.) (Jacq.) B.K.Simon \& S.W.L.Jacobs] foram registradas na região.

\section{Discussão}

O número de espécies registradas representa 22 e 67\%, respectivamente, da riqueza amostrada nas Restingas de Sergipe (Oliveira et al. 2014) e da Reserva Biológica (REBIO) de Santa Isabel (Oliveira et al. 2015). De forma geral, a riqueza registrada foi inferior à observada na maioria das áreas de Restinga já estudadas na região Nordeste (Tabela S1; disponível como material suplementar <https://figshare.com/s/029b4ee4cd43daded4c8>).

Diversas pesquisas têm confirmado a expressiva riqueza da família Fabaceae nas Restingas Nordestinas (ca. de 70\% dos estudos avaliados, ver Tab. S1). Em Sergipe, até o momento, esta família aparece como a de maior riqueza em áreas de Restinga (Oliveira et al. 2014; Oliveira et al. 2015). As espécies dessa família são muito adaptadas a colonizar a ambientes praticamente estéreis, como a areia (Carvalho e Oliveira-Filho 1993), provavelmente pela associação com bactérias fixadoras de nitrogênio em nódulos em suas raízes (Queiroz 2009).

Cyperus é citado como um dos gêneros mais importantes com relação ao número de espécies em áreas de Restinga da costa nordestina no Ceará (Santos-Filho et al. 2011), Pernambuco (Almeida-Jr et al. 2007) e Bahia (Queiroz et al. 2012). As espécies deste gênero são muito comuns em lagoas e brejos ao longo do litoral brasileiro, sendo encontrados registros no Pará (Amaral et al. 2008), Sergipe (Oliveira e Landim 2014), Espírito Santo (Valadares et al. 2011) e Rio de Janeiro (Paz e Bove 2007). Na área de estudo, dentre as espécies de Cyperus, apenas $C$. articulatus foi coletada em área inundável, o que pode ser decorrência da escassez de lagoas e brejos nas Restingas da ZEU, como resultado do processo de ocupação e urbanização dessas áreas. Esse resultado sugere o maior risco de extinção local de espécies hidrófitas em áreas de Restinga sujeitas a aterros para a construção civil.

Variações na riqueza de plantas nas Restingas brasileiras têm sido associadas a características edáficas e variações no regime de inundação do substrato (Cantarelli et al. 2012; Magnago et al. 2012; Marques et al. 2015). Condições edáficas desfavoráveis podem representar filtros bióticos para o estabelecimento de plantas nos habitats de Restinga, limitando a riqueza de espécies (Fernandes e Queiroz 2015).

Considerando os estudos que utilizaram o mesmo sistema de classificação (Silva e Britez 2005) adotado no presente trabalho, os tipos fitofisionômicos encontrados na ZEU de Aracaju são similares àqueles observados em Restingas da região Nordeste, incluindo áreas no Maranhão (Lima et al. 2017), Piauí (Santos-Filho et al. 2010) e Sergipe (Oliveira e Landim 2014; Dantas et al. 2019). Entre as fitofisionomias descritas por Silva e Britez (2005), 
seis não foram registrados na ZEU de Aracaju ("campo fechado inundável”, "campo aberto inundável”, "campo aberto inundável halófilo", "fruticeto aberto inundável", "fruticeto fechado inundável” e "floresta inundável”).

Considerando que as fitofisionomias campestres foram as mais encontradas ao longo da ZEU, era esperado que as ervas tenham sido a forma de vida mais expressiva em relação ao número de espécies. Na realidade, estes resultados podem ser também um reflexo do estágio de degradação, mesmo que em estágios de regeneração, da maior parte das áreas de restinga da ZEU. Espécies arbóreas são mais visadas para a extração de lenha e madeira para a construção de cercas e casas, além de apresentarem regeneração mais lenta que o estrato herbáceo (Fávero e Pavan 1997; Chazdon 2012).

É provável que a proximidade seja um dos fatores que contribuíram para ao grupamento observado entre as áreas de restinga analisadas. A maior parte dos estudos encontrados sobre essa tipologia vegetal tem ressaltado o papel da distância geográfica na determinação de padrões de similaridade florística (e.g., Assis et al. 2004; Guedes et al. 2006; Silva et al. 2008).

Embora seja notável a influência da proximidade das áreas sobre a sua composição de espécies, o papel de fatores ecológicos e históricos não deve ser completamente desconsiderado. A similaridade florística entre áreas de Restinga pode estar também relacionada às características ambientais de cada área, como clima, solo e níveis de antropização (Assis et al. 2004; Silva et al. 2008; Assis et al. 2011; Almeida-Jr et al. 2016). Além disso, fatores históricos, como o isolamento causado por barreiras naturais (e.g., grandes rios), podem interromper a dispersão entre áreas, modificando a estrutura genética das espécies, levando, eventualmente, à especiação (Ricklefs e Relyea 2016). Nesse sentido, o isolamento do sub-grupo 2.3 (AL) dos demais pode estar relacionado à atuação do rio São Francisco como uma barreira, ao menos para determinados grupos (e.g., Landim et al. 2015).

As diferenças de distinção taxonômica observadas podem estar, também, associadas ao histórico de perturbação entre as áreas analisadas. O índice de distinção taxonômica é muito sensível a perturbações ambientais, sendo seus menores valores registrados em ambientes degradados (Warwick e Clarke 1995; Warwick e Clarke 1998). Dentre as áreas selecionadas para essa análise, as Restingas da ZEU de Aracaju parecem ser aquelas com o pior estado de conservação, onde a antropização pode já ter extinguido localmente alguns grupos de plantas. Por exemplo, a fitofisionomia florestal, praticamente ausente na ZEU de Aracaju, é habitat para várias espécies epífitas e trepadeiras. A ausência desses grupos na flora da ZEU de Aracaju pode ter contribuído para a sua baixa distinção taxonômica (ver Moreno et al. 2009). Assim, a antropização na região estudada parece estar originando um processo de homogeneização biótica (McKinney 2006), contribuindo para que as espécies sejam muito relacionadas em baixos níveis taxonômicos (Moreno et al. 2009).

Nesse sentido, a menor riqueza observada para as Restingas estudadas em relação às áreas dessa vegetação no litoral norte do estado (REBIO de Santa Isabel) é, muito provavelmente, resultado da falta de proteção legal (delimitação de áreas como unidades de conservação) e efetiva (fiscalização regular e coibição das inúmeras e frequentes agressões) do que um padrão natural de distribuição da biodiversidade nativa.

Vários dos pontos de amostragem apresentaram áreas de degradação circundando as manchas de vegetação nativa onde as coletas foram realizadas. A presença dos impactos antrópicos, tais como acúmulo de lixo, queimadas, desmatamento, criação de gado e a presença de construções irregulares registrados pelos autores nas áreas de coleta, contribuem para a diminuição da sua diversidade. A intensidade da ocupação urbana e a ausência de planejamento na ZEU de Aracaju resultaram em modificações na paisagem natural, tendo como consequência diversos impactos ambientais (Oliveira e Andrade 2015).

O efeito da antropização nas Restingas da ZEU se mostra, também, pela presença de espécies exóticas invasoras, causa de preocupação no tocante à efetividade de conservação da diversidade nativa nas áreas remanescentes (Delariva e Agostinho 1999). Enquanto Nymphoides bumboldtiana (Kunh) Kuntze tem sido reconhecida por alterar as condições ambientais de ecossistemas aquáticos (e.g., na incidência de luz e disponibilidade de oxigênio), Megathyrsus maximus (Jacq.) (Jacq.) B.K.Simon \& S.W.L.Jacobs, a gramínea invasora conhecida como "capimcolonião”, pode acumular biomassa rapidamente e é muito resistente ao fogo (I3N Brasil/Instituto Hórus 2018). 
Ambas as espécies, através de competição, podem comprometer a sobrevivência de espécies nativas (I3N Brasil/ Instituto Hórus 2018).

Deve ser registrada a ausência de Hancornia speciosa Gomes (Mangabeira) nas áreas de Restingas analisadas na ZEU. Esta espécie possui grande importância social, econômica e cultural no estado de Sergipe (Soares et al. 2016). Neste estado, o extrativismo do fruto da Mangabeira ocorre em populações naturais, distribuídas ao sul (municípios de Itaporanga D’Ajuda, Estância, Indiaroba e Santa Luzia do Itanhy) e ao norte da ZEU de Aracaju (municípios de Barra dos Coqueiros, Pirambu, Pacatuba, Japaratuba, Neópolis e Brejo Grande) (Soares et al. 2016). Na ZEU de Aracaju, H. speciosa foi observada apenas em um remanescente de Restinga em área à qual não foi possível o acesso por estar cercada, não tendo sido, portanto, coletada. Este fato, somado à incerteza de que esses indivíduos não representem uma população nativa, mas, ao contrário, tenham sido plantados, contribuíram para a sua não inclusão na listagem florística compilada.

As Restingas da ZEU de Aracaju, embora atualmente ameaçadas pelas atividades antrópicas, representam áreas importantes para a conservação da biodiversidade, e, por sua vez para a manutenção dos seus serviços ambientais. Os resultados observados, como a relativamente baixa, mas relevante, diversidade e os registros de novas ocorrências para as Restingas de Sergipe, reforçam o papel das Restingas da ZEU de Aracaju na conservação da diversidade regional. Embora o foco do presente trabalho seja a diversidade florística, não se pode ignorar que essas áreas são habitat, e suas espécies, recursos, para os elementos da fauna local, igualmente ameaçada pelo processo de ocupação crescente e desordenada dessas Restingas. A inexistência de áreas protegidas na região e as agressões observadas a áreas que seriam de preservação permanente tornam prioritárias ações de conservação e gestão, incluindo maiores investimentos no planejamento do uso do solo, a criação de, ao menos uma, unidade de conservação na região, assim como a implantação de programas permanentes de educação ambiental e fiscalizações mais efetivas, visando minimizar e controlar os danos causados pela ação humana.

\section{Conclusão}

O estudo revelou que vegetação de Restinga da ZEU de Aracaju se encontra sob forte influência da crescente antropização, observada, por exemplo, na frequência de suas fitofisionomias, dentre as quais formações florestais são praticamente inexistentes. A despeito disso, foram feitos registros de novas ocorrências para as Restingas de Sergipe. A análise de similaridade mostrou um papel importante da distância geográfica na similaridade florística entre as áreas de Restinga analisadas, embora não seja descartada a influência de fatores ecológicos e históricos. Os valores de distinção taxonômica obtidos reforçam a avaliação do estado de intensa antropização observada in loco nessas Restingas. A inexistência de unidades de conservação (UCs) nessa região, somada às graves perturbações e ameaças antrópicas observadas ressaltam a necessidade de ações visando à preservação dessa região, como o estabelecimento de UCs, a clara delimitação e identificação das áreas de preservação permanente (APPs), a implantação de programas permanentes de educação ambiental e a mais efetiva fiscalização das infrações.

\section{Agradecimentos}

À Universidade Federal de Sergipe (UFS), pela concessão de transporte para as campanhas de campo. À Eladio dos Santos e à Bióloga Elânia dos Santos Ferreira-Sobrinho, pelo auxílio nos trabalhos de campo. 


\section{REFERÊNCIAS}

Almeida-Jr EB, Pimentel RMM, Zickel CS. 2007. Flora e formas de vida em uma área de restinga no litoral Norte de Pernambuco, Brasil. Revista de Geografia, 24(1): 19-34.

Almeida-Jr EB, Machado MA, Medeiros DPW, Pinheiro TS, Zickel CS. 2016. Florística de uma área de vegetação de influência marinha no litoral sul de Alagoas, Brasil. Revista Brasileira de Geografia Física, 9(5): 1400-1409.

Alvares CA, Stape JL, Sentelhas PC, Gonçalves JLM, Sparovek G. 2014. Köppen’s climate classification map for Brazil. Meteorologische Zeitschrift, 22(6): 711-728. https://doi.org/10.1127/0941-2948/2013/0507

Amaral DD, Prost MT, Bastos MNC, Costa-Neto SV, Santos JUM. 2008. Restingas do litoral amazônico, estados do Pará e Amapá, Brasil. Boletim do Museu Paraense Emílio Goeldi, 3(1): 35-67.

APG IV - Angiosperm Phylogeny Group IV. 2016. An update of the Angiosperm Phylogeny Group Classification for the orders and families of flowering plants: APG IV. Botanical Journal of the Linnean Society, 181: 1-20. https://doi. org/10.1111/boj.12385

Aracaju. 1982. Lei no 873/82 de 01 de outubro de 1982. Estabelece nova delimitação dos Bairros de Aracaju e dá outras providências correlatas. Disponível em: <https://www.aracaju.se.gov.br/userfiles/emurb/2011/08/Diversas_Lei0873_1982_ DelimitacaoBairrosAracaju.pdf $>$. Acesso em: 10 nov. 2019.

Araújo DSD, Henriques RPB. 1984. Análise florística das restingas do Estado do Rio de Janeiro. In: Lacerda LD et al. (Orgs), Restingas: origem, estrutura e processos, Niterói: CEUFF, p. 159-193.

Araujo DSD, Pereira MCA. 2009. Sandy coastal vegetation. In: Del Claro K et al. (Eds), Encyclopedia of Life Support Systems (EOLSS): tropical biology and conservation management - vol. IV, Oxford: EOLSS-UNESCO, p. 173-189.

Assis AM, Thomaz LD, Pereira OJ. 2004. Florística de um trecho de floresta de restinga no município de Guarapari, Espírito Santo, Brasil. Acta Botanica Brasilica, 18(1): 191-201. http://dx.doi.org/10.1590/S0102-33062004000100016

Assis MA, Prata BEM, Pedroni F, Sanchez M, Eisenlohr PV, Martins FR, Santos FAM, Tamashiro JY, Alves LF, Vieira SA, Piccolo MC, Martins SC, Camargo PB, Carmo JB, Simões E, Martinelli LA, Joly CA. 2011. Restinga and Lowland forests in coastal plain of southeastern Brazil: vegetation and environmental heterogeneity. Biota Neotropica, 11(2). http://dx.doi. org/10.1590/S1676-06032011000200012.

BFG - The Brazil Flora Group. 2018. Brazilian Flora 2020: Innovation and collaboration to meet Target 1 of the Global Strategy for Plant Conservation (GSPC). Rodriguésia, 69(4): 1513-1527.http://dx.doi.org/10.1590/2175-7860201869402

Boyle B, Hopkins N, Lu Z, Garay JAR, Mozzherin D, Rees T, Matasci N, Narro ML, Piel WH, Mckay JS, Lowry S, Freeland C, Peet RK, Enquist BJ. 2013. The taxonomic name resolution service: an online tool for automated standardization of plant names. BMC Bioinformatics, 14(16). https://doi.org/10.1186/1471-2105-14-16

Brasil. 2006. Lei no 11.428/2006. Disponível em: <http://www.planalto.gov.br/ccivil_03/_ato2004-2006/2006/lei/111428. htm>. Acesso em: 09 nov. 2019.

Brasil. 2012. Lei no 12.651/2012. Disponível em: <http://www.planalto.gov.br/ccivil_03/_Ato2011-2014/2012/Lei/L12651. htm>. Acesso em: 09 nov. 2019. 
Brito CI, Queiroz LP, Guedes MLS, Oliveira NC, Silva LB. 1993. Flora fanerogâmica das dunas e lagoas do Abaeté, Salvador, Bahia. Sitientibus, 11: 31-46.

Cantarelli JRR, Almeida-Jr EB, Santos-Filho FS, Zickel CS. 2012. Tipos fitofisionômicos e florística da restinga da APA de Guadalupe, Pernambuco, Brasil. Insula, 41: 95-117.

Carvalho DA, Oliveira-Filho AT. 1993. Avaliação da recomposição da cobertura vegetal de dunas de rejeito de mineração, em Mataracá/PB. Acta Botanica Brasilica, 7(2): 107-117. http://dx.doi.org/10.1590/S0102-33061993000200006.

Chazdon R. 2012. Regeneração de florestas tropicais. Boletim do Museu Paraense Emílio Goeldi, 7(3): 195-218.

Cordazzo CV, Paiva JB, Seeliger U. 2006. Guia ilustrado: plantas das dunas da costa sudoeste Atlântica, Pelotas: USEB, $107 \mathrm{p}$.

CRIA - Centro de Referência em Informação Ambiental. 2017. SpeciesLink. Disponível em: <http://splink.cria.org.br/>. Acesso em 11 jan. 2017.

Dantas TVP, Fortes FP, Ribeiro AS. 2019. Mapeamento e caracterização fitofisionômica da planície costeira do litoral norte do estado de Sergipe. Revista Brasileira de Geografia Física, 12(3): 1171-1179.

Delariva RL, Agostinho AA. 1999. Introdução de espécies: uma síntese comentada. Acta Scientiarum Biological Sciences, 21(2): 255-262. https://doi.org/10.4025/actascibiolsci.v21i0.443

ESRI. 2008. ArcGIS 9.3. New York.

Falkenberg DB. 1999. Aspectos da flora e da vegetação secundária da restinga de Santa Catarina, Sul do Brasil. Insula, 28: $1-30$.

Fávero AO, Pavan S. 1997. Botânica Econômica, São Paulo: Catálise Editora, 91 p.

Fernandes MF, Queiroz LP. 2015. Floristic surveys of Restinga Forests in southern Bahia, Brazil, reveal the effects of geography on community composition. Rodriguésia, 66(1): 51-73. http://dx.doi.org/10.1590/2175-7860201566104

Giulietti AM, Rapini A, Andrade MJG, Queiroz LP, Silva JMC. 2009. Plantas raras do Brasil, Belo Horizonte: Conservação Internacional e Universidade Estadual de Feira de Santana, 498 p.

Gomes FS, Guedes MLS. 2014. Flora vascular e formas de vida das formações de restinga do litoral norte da Bahia, Brasil. Acta Biológica Catarinense, 1(1): 22-43. http://dx.doi.org/10.21726/abc.v1i1.82

Guedes D, Barbosa LM, Martins SE. 2006. Composição florística e estrutura fitossociológica de dois fragmentos de floresta de restinga no Município de Bertioga, SP, Brasil. Acta Botanica Brasilica, 20(2): 299-311. http://dx.doi.org/10.1590/ S0102-33062006000200006

Hammer Ø, Harper DAT, Ryan PD. 2013. PAST - Palaeontological statistics. Disponível em: <http://folk.uio.no/ohammer/ past/>. Acesso: 10 jun. 2017.

Holzer W, Crichyno J, Pires AC. 2004. Sustentabilidade da urbanização em áreas de restinga: uma proposta de avaliação pós-ocupação. Paisagem ambiente, 19: 49-65. 
I3N Brasil/Instituto Hórus. 2018. Base de dados nacional de espécies exóticas invasoras. Disponível em: <http://i3n. institutohorus.org.br/www>. Acesso em: 10 jan. 2018.

Lacerda LD, Araújo DSD, Maciel NC. 1993. Drycoastal ecosystems of the tropical Brazilian coast. In: Maarel E van der (Ed), Dry Coastal-ecosystems: Africa, Asia, Oceania, Amsterdam: Elsevier, p. 477-493.

Landim MF, Proença CEB, Sales AB, Matos IS. 2015. Floristic characterization of an Atlantic Rainforest remnant in Southern Sergipe: Crasto forest. Biota Neotropica 15: 1-16. https://doi.org/10.1590/1676-06032014003613

Lima GP, Lacerda DMA, Lima HG, Almeida-Jr EB. 2017. Caracterização fisionômica da Restinga da Praia de Panaquatira, São José de Ribamar, Maranhão. Revista Brasileira de Geografia Física, 10(6): 1910-1920.

Magnago LFS, Martins SV, Schaefer CEGR, Neri AV. 2012. Restinga forests of the Brazilian coast: richness and abundance of tree species on different soils. Anais da Academia Brasileira de Ciências, 84(3): 807-822. http://dx.doi.org/10.1590/ S0001-37652012000300023

Marques MCM, Silva SM, Liebsch D. 2015. Coastal plain forests in southern and southeastern Brazil: ecological drivers, floristic patterns and conservation status. Brazilian Journal of Botany, 38: 1-18. https://doi.org/10.1007/s40415-015$0132-3$

Martinelli M, Moraes MA. 2013. Livro Vermelho da Flora do Brasil, Rio de Janeiro: Instituto de Pesquisas Jardim Botânico do Rio de Janeiro e CNC Flora, 1102 p.

Martins SE, Rossi L, Sampaio PSP, Magenta MAG. 2008. Caracterização florística de comunidades vegetais de restinga em Bertioga, SP, Brasil. Acta Botanica Brasilica, 22(1): 249-274. http://dx.doi.org/10.1590/S0102-33062008000100024.

McKinney M. 2006. Urbanization as a major cause of biotic homogenization. Biological Conservation, 127: 247-260. https://doi.org/10.1016/j.biocon.2005.09.005

Meyer C, Weigelt P, Kreft H. 2016. Multidimensional biases, gaps and uncertainties in global plant occurrence information. Ecology Letters, 19: 992-1006. https://doi.org/10.1111/ele.12624

Moraes CAR. 2007. Contribuição para a gestão da zona costeira do Brasil: elementos para uma geografia do litoral brasileiro, São Paulo: Annablume, 232 p.

Moreno CE, Castillo-Campos G, Verdú JR. 2009. Taxonomic diversity as complementary information to assess plant species diversity in secondary vegetation and primary tropical deciduous forest. Journal of Vegetation Science, 20: 935943. https://doi.org/10.1111/j.1654-1103.2009.01094.x

Mori AS, Silva LAM, Lisboa G, Coradin L. 1985. Manual de manejo do herbário fanerogâmico, Ilhéus: Centro de Pesquisas do Cacau, 84 p.

Oliveira LS, Andrade, ACS. 2012. Dinâmica da paisagem da Zona de Expansão de Aracaju-SE. Scientia Plena, 8.

Oliveira LS, Andrade ACS. 2015. Inserção antrópica no modelamento da paisagem costeira da zona de expansão do município de Aracaju/SE. Revista Brasileira de Geografia Física, 8(2): 391-401.

Oliveira EVS, Ferreira-Sobrinho ES, Landim MF. 2015. Flora from the restingas of Santa Isabel Biological Reserve, northern coast of Sergipe state, Brazil. Check List, 11. http://dx.doi.org/10.15560/11.5.1779 
Oliveira EVS, Landim MF. 2014. Caracterização fitofisionômica das restingas da Reserva Biológica de Santa Isabel, litoral Norte de Sergipe. Scientia Plena, 10(10): 1-10.

Oliveira EVS, Landim MF. 2016. Flora das Restingas de Sergipe: padrões de distribuição espacial e status de conservação de suas espécies. Natureza on line, 14(1): 23-31.

Oliveira EVS, Lima JF, Silva TC, Landim MF. 2014. Checklist of the flora of the Restingas of Sergipe State, Northeast Brazil. Check List, 10: 529-549. http://dx.doi.org/10.15560/10.3.529

Paz J, Bove CP. 2007. Hidrófitas vasculares da lagoa de Carapebus, Parque Nacional da Restinga de Jurubatiba, Rio de Janeiro, Brasil. Revista Brasileira de Biociências, 5: 495-497.

Pereira OJ, Araújo DSD. 2000. Análise florística das restingas dos estados do Espírito Santo e Rio de Janeiro. In: Esteves FA, Lacerda LD (Eds), Ecologia de restingas e lagoas costeiras, Macaé: NUPEM/UFRJ, p. 25-63.

Pergentino TC, Landim MF. 2014. Restingas de Sergipe: contribuição ao conhecimento da sua composição florística e análise sobre o status de conservação atual. In: Landim M, Guimarães CP (Orgs), Ecologia, uso potencial e conservação de ecossistemas costeiros sergipanos, São Cristóvão: Editora UFS, p. 103-128.

Pinto-Sobrinho FA, Souza CRG, Mogollón JEJB. 2011. Análise estrutural de florestas de restinga associadas a depósitos marinhos pleistocênicos e holocênicos na bacia do rio Itaguaré, Bertioga (SP). Revista do Instituto Geológico, 32: 27-40.

Prata APN, Amaral MCE Farias MCV, Alves MV (Orgs). 2013. Flora de Sergipe (volume 1), Aracaju: Gráfica e Editora Triunfo, 299 p.

Queiroz LP. 2009. Leguminosas da caatinga, Feira de Santana: UEFS, 443 p.

Queiroz EP, Cardoso DBOS, Ferreira MHS. 2012. Composição florística da vegetação de restinga da APA Rio Capivara, Litoral Norte da Bahia, Brasil. Sitientibus, 12(1): 119-141.

Rambo, B. 1954. História da flora do litoral riograndense. Sellowia, 6(6): 113-172.

Reitz, R. 1961. A vegetação marítima de Santa Catarina. Sellowiana, 9(8): 20-70.

Ricklefs R, Relyea R. 2016. A economia da natureza, Rio de Janeiro: Guanabara Koogan, 807 p.

Sacramento ACS, Almeida-Jr EB, Zickel CS. 2007. Aspectos florísticos da vegetação de restinga no litoral de Pernambuco. Revista Árvore, 31(6): 1121-1130. http://dx.doi.org/10.1590/S0100-67622007000600017.

Santos-Filho FS, Almeida-Jr EB, Soares CJRS, Zickel CS. 2010. Fisionomias das restingas do Delta do Parnaíba, Nordeste, Brasil. Revista Brasileira de Geografia Física, 3: 218-227.

Santos-Filho FS, Almeida-Jr EB, Bezerra LF, Lima LF, Zickel CS. 2011. Magnoliophyta, restinga vegetation, state of Ceará, Brazil. Check List, 7(4): 478-485. http://dx.doi.org/10.15560/7.4.478

Santos-Neto AM, Oliveira EVS, Faiad PJB, Landim MF. 2018. Sazonalidade e estrutura da vegetação herbácea de Restingas: uma análise na Reserva Biológica de Santa Isabel, SE. Brazilian Journal of Ecology, 1: 5-21. 
Scherer A, Maraschin-Silva F, Baptista LRM. 2005. Florística e estrutura do componente arbóreo de matas de restinga arenosa no Parque Estadual de Itapuã, RS, Brasil. Acta Botanica Brasílica, 19(4): 717-726. http://dx.doi.org/10.1590/ S0102-33062005000400006.

Secretaria de Estado do Planejamento e da Ciência e Tecnologia - SEPLANTEC. 1997. Perfis Municipais - Aracaju, 75 p. Silva SM, Britez RM. 2005. A vegetação da planície costeira. In: Marques MCM, Britez RM (Orgs), História natural e conservação da ilha do Mel, Curitiba: Editora da UFPR, p. 49-84.

Silva SSL, Zickel CS, Cestaro LA. 2008. Flora vascular e perfil fisionômico de uma restinga no litoral sul de Pernambuco. Acta Botanica Brasilica, 22: 1123-1135. http://dx.doi.org/10.1590/S0102-33062008000400023.

Soares ANR, Vitória MF, Nascimento ALS, Ledo AS, Rabbani ARC, Silva AVC. 2016. Genetic diversity in natural populations of Mangaba in Sergipe, the largest producer State in Brazil. Genetics and Molecular Research, 15(3). http://10.4238/gmr.15038624.

Suguio K, Tessler MG. 1984. Planícies de cordões litorâneos quaternários do Brasil: origem e nomenclatura. In: Lacerda LD et al. (Orgs), Restingas: Origem, estrutura e processos, Niterói: Universidade Federal Fluminense/CEUFF, 15-25 p.

Valadares RT, Souza FBC, Castro NGD, Peres ALSS, Schneider SZ, Martins MLL. 2011. Levantamento florístico de um brejo-herbáceo localizado na restinga de Morada do Sol, município de Vila Velha, Espírito Santo, Brasil. Rodriguésia, 62(4): 827-834. http://dx.doi.org/10.1590/S2175-78602011000400010.

Villwock JE, Lessa GC, Suguio K, Angulo RJ \& Dillenburg DR. 2005. Geologia e geomorfologia de regiões costeiras. In: Souza CRG et al. (Eds), Quaternário do Brasil, Ribeirão Preto: Editora Holos, p. 94-107.

Warwick RM, Clarke KR. 1995. New 'biodiversity' measures reveal a decrease in taxonomic distinctness with increasing stress. Marine Ecology Progress Series, 129: 301-305.

Warwick RM, Clarke KR. 1998. Taxonomic distinctness and environmental assessment. Journal of Applied Ecology, 35 : 532-543.

Zar JH. 2010. Biostatistical analysis, New Jersey: Prentice-Hall, 944 p.

Zickel CS, Almeida-Jr EB, Medeiros DPW, Lima PB, Souza TMS, Lima AB. 2007. Magnoliophyta species of restinga, State of Pernambuco, Brazil. Check List, 3(2): 224-241.http://dx.doi.org/10.15560/3.3.224 